\title{
Near-wall and Turbulence Behavior of Swirl Flows through an Aerodynamic Nozzle
}

\author{
Md. Tanvir Khan*, Sharif M. Islam, and Zahir U. Ahmed \\ Department of Mechanical Engineering, Khulna University of Engineering \& Technology, Khulna-9203, BANGLADESH
}

Received: June 04, 2020, Revised: June 21, 2020, Accepted: June 21, 2020, Available Online: June 28, 2020

\begin{abstract}
It is often a challenge to achieve uniform flow in turbulent swirl flow and to predict the flow within the nozzle as measurement diagnostics face difficulty to capture both mean flow and turbulence. The purpose of this study is to numerically investigate the near wall flow characteristics and turbulent behavior for the effect of different tangential inlet numbers of an incompressible turbulent swirl air jet. In this regard, axial-plus-tangential flow based swirling nozzle is considered for the simulation using finite volume method, where turbulence is approximated by the Shear Stress Transport (SST) $k-\omega$ model. The results show that axial and tangential velocity at the wall vicinity response the most. Moreover, the turbulent flow characteristic for no swirl flow is nearly uniform, but for swirl flow it fluctuates abruptly near the inlet section where the swirl has introduced. The skin friction coefficient for 2TP is the maximum for swirl flow and for no swirl condition the skin friction coefficient is nearly uniform. Due to the swirl introduction the pressure drop characteristics near the nozzle center response quickly and near the wall vicinity this property changes slowly. The magnitude of swirl decay fluctuates before the nozzle converging section however after the nozzle converging section the swirl decay is nearly constant. The local swirl near the inlet is highly unpredictable although after the nozzle converging section the local swirl profile is nearly similar for 2TP, 3TP and 4TP.
\end{abstract}

Keywords: Turbulent; Nozzle; Swirl; Aerodynamic; CFD; Pressure.

This work is licensed under a Creative Commons Attribution-NonCommercial 4.0 International

\section{Introduction}

Swirl flows are a classical form of fluid flow and typically encountered in many engineering applications, such as gas turbine combustor and cyclonic separator, as well as in nature, such as tornedo. In swirling flows generally two types of vortex emerge, namely solid body rotation and free vortex flow. However, in actual practice, a mixed of these two typically appears whereby azimuthal velocity increases with radius in the core and decreases afterwards. The academic and industrial research on swirling jets was found to be challenging due to their unique characteristics, such as flow reversal, vortex bubble and anisotropic turbulences in both free and impinging jets. The detailed flow physics, instability and above features of swirling flows are explicitly available in the literature [1]. The axial and radial pressure gradient in swirling flows affects downstream flow development and wall-bounded flows. Swirl flows are generated in different applications in many ways, such as rotating pipe, rotating vanes and twisted tapes inside stationary pipe, axial-plus-tangential entry [2]-[7]. The wide variations of generations can be divided into two broad groups: geometrical and aerodynamical. In geometrically generated swirl flows, for example rotating vanes or twisted tapes inside pipes, the flow is disturbed by such geometries or obstacles, which exacerbates the generality of the flow and difficult to draw a summative observation. On the other hand, aerodynamically generated swirl flows, such as rotating pipe or axial-plus-tangential entry flow, have better control of the flow and common flow physics may establish in different researches. Despite geometrically generated swirl flows are widely available in the literature and still progressing, fundamentals of aerodynamic swirl flows are currently being investigated in detail because of its prevalence to use recently in number heat treatment applications.
Yajnik and Subbaiah [8] experimentally studied the effects of swirl on internal turbulent flows by conducting experiments on turbulent pipe flow. They found that the mean velocity profiles close to and away from the wall admit similarity representations at sufficiently large Reynolds numbers and extended velocity defect law is sensitive to swirl as the wall law is not sensibly dependent on swirl. Chang and Dhir [9] found a flow reversal region in axial velocity profile in the central portion of an acrylic tube and an axial velocity increase near the wall. Kito and Kato [10] studied the near wall velocity distribution of turbulent swirling flow in circular pipes and concluded that the flow becomes three-dimensional after transitional swirl intensity. Later, Kitoh [11] investigated the turbulent behavior of freevortex-type swirling flow through a long straight circular pipe and reported that the swirling component decays downstream as a result of wall friction. Buschmann et al. [12] explored the wall behavior, the location of the peak Reynolds shear stresses and the three normal stresses of turbulent channel/pipe flows, and stated that no scaling works equally for all parameters. Ahmed et al. [13] conducted measurements of mean velocity and turbulence of swirling flows using dual wire CTA probe both in the core and near-wall regions. However, the measurements were confined to the immediately above the nozzle exit plane. Effect of viscosity and surface tension of fluids and associated instabilities in annular flow were also analyzed in some studies [14]-[15].

Since both DNS and LES typically require sufficiently smaller sized mesh or large mesh quantity to resolve small-scale turbulence, Reynolds Averaged Navier-Stokes (RANS) were widely used in swirling flows by many investigators. Although it is believed that among various RANS models the Reynolds Stress Model (RSM) would perform better because of its ability to capture anisotropy of turbulent shear stress, this hypothesis 
was not found to be universal in the past [16]-[17]. In fact, some studies revealed that two-equation models (based on Boussinesq approximation) can be applied with acceptable accuracy in comparison with experimental data for moderate swirl flows [18]-[19]. Nouri-Borujerdi and Kebriaee [20] simulated the turbulent boundary layer of an incompressible viscous swirling flow through a conical chamber using finite volume method and reported that the boundary layer thickness is dependent on the velocity ratios, Reynolds number and nozzle angle. Najafi et al. [21] investigated turbulent swirling decay in a vertical straight fixed pipe where swirl in induced by rotating honeycombs. They showed RSM with two-layer zone model for different near wall approaches are fairly well to predict the swirling flow but fail to predict the pressure distribution along the pipe wall. Islam et al. simulated an aerodynamically generated swirl flow using SST k$\omega$ model in the nozzle exit plane. The result showed centerline velocity [22] decay for introducing low levels of swirl into the impinging jet and a significant reduction in turbulent kinetic energy at the wall region. More recently, researchers [23]-[27] performed simulations of aerodynamic swirl flow using SST k$\omega$ model and validated their results with the literature.

The above discussion revealed that although a significant amount of research available on swirling flows, they are mostly confined to gas turbine combustors, cyclonic separator, or geometry induced swirl flows. Relevant aerodynamically swirling flows are either classical in nature (experimental) with outdated experimental facility to resolve near-wall and turbulence behaviors inside the nozzle or tested robustness of various numerical schemes against experimental data with limited focus on flow behaviors. Thus, a detailed study for the effect of geometric parameters on near-wall and turbulence behaviors for an aerodynamic swirl nozzle appears to be limited in the literature. As such, the current numerical study will bridge this gap by investigating non-swirling and swirling flows from an aerodynamic nozzle for the same initial and flow conditions. The paper will examine the effect of number of tangential ports on mean and turbulence flow development along the length of the nozzle.

\section{Methodology}

\subsection{Problem formulation}

An aerodynamically generated swirl nozzle, which is capable of seamless transition from non-swirling to highly swirling jets, is considered in this study. The nozzle is axialplus-tangential entry type and consists of three tangential around the nozzle periphery and an axial port at the bottom of the nozzle. Detailed dimension of this nozzle is available in [4],[28], hence is not repeated here for brevity. The uniform flow was ensured by the flow settling chamber with honeycomb. The aerodynamic swirl flow was generated when both the uniform axial and tangential flows from different number of circumferentially oriented and inclined ports mix together farther downstream. In this case, three variants of tangential ports: 2-Tangential ports (2TP), 3-Tangential ports (3TP) and 4-Tangential ports (4TP) are considered. The exit of the nozzle has diameter $(D) 40 \mathrm{~mm}$, with a total length of 577 $\mathrm{mm}$. A three-dimensional view with the relative orientation of tangential ports is shown in Fig. 1.

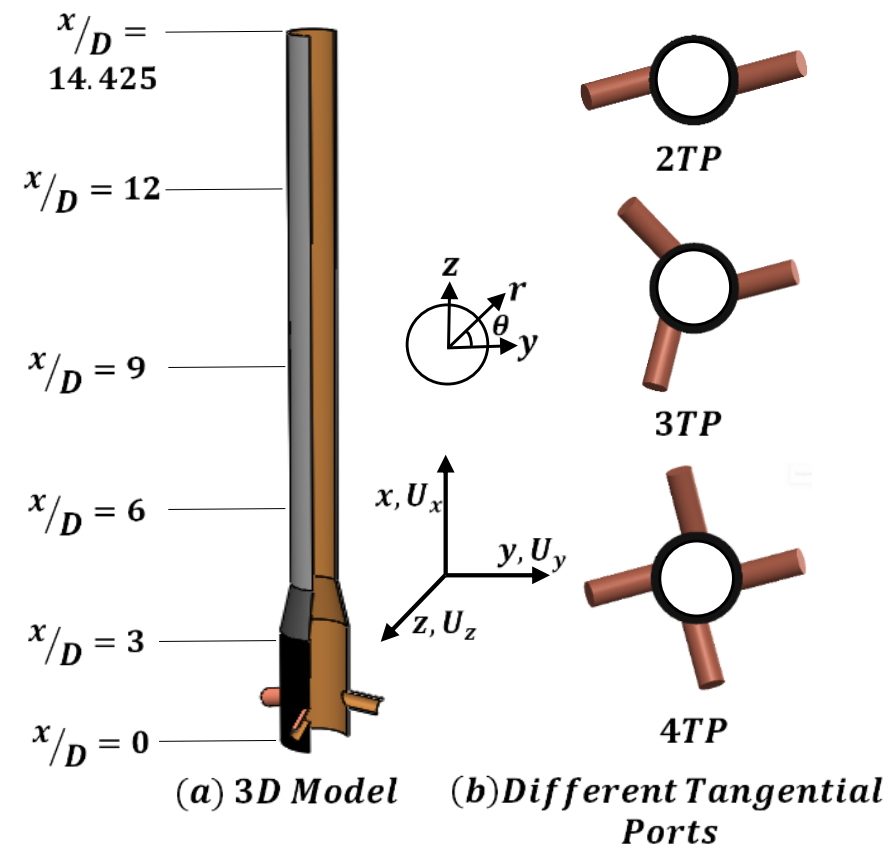

Fig. 1 (a) CAD view of the aerodynamic swirl nozzle (sliced to show the internal cavity), and (b): different number of tangential ports to impart azimuthal component.

\subsection{Governing Equation and Boundary Conditions}

The governing equations to solve the incompressible, steady-state flow characteristics within the aerodynamic swirl nozzle are the conservation of mass and momentum as follows: $\nabla \cdot \overrightarrow{\hat{V}}=0$

$\rho(\overrightarrow{\hat{V}} \cdot \nabla) \overrightarrow{\hat{V}}=-\nabla \hat{P}+\mu \nabla^{2} \overrightarrow{\hat{V}}$

Here, $\overrightarrow{\hat{V}}$ is the velocity vector, and $\rho$ and $\mu$ are the density and dynamic viscosity of the fluid, respectively. Since the current problem is turbulent in nature, RANS approach is applied to solve the mean and turbulence quantities. In RANS approximation, each variable is composed of time-averaged part (steady) and turbulence part, as shown below:

$\hat{\phi}=\phi+\phi^{\prime}$

where $\phi$ is a variable used in equations (1) and (2). Upon implementing the equation (3) into the governing equations and using equation (1) and setting time-average of turbulence equals to zero, the resulting RANS equations emerge. The RANS equations are similar to the governing equations, except an additional term $\left(\overline{\rho u_{\imath}^{\prime} u_{\jmath}^{\prime}}\right)$ in equation (2). This additional term is known as turbulent shear stress, which governs the turbulence characteristics. The shear stress components are determined via mean velocity gradients by the Boussinesq hypothesis:

$$
\overline{u_{i}^{\prime} u_{j}^{\prime}}=\frac{2}{3} k \delta_{i j}-\mu_{t}\left(\frac{\partial u_{i}}{\partial x_{j}}+\frac{\partial u_{j}}{\partial x_{i}}\right)
$$


Where, $\mu_{t}$ is the turbulent viscosity and is a function of $k$ and $\omega$, which are determined via an appropriate turbulence model. In this research, the SST k- $\omega$ model, proposed by Menter and Egorov [29], is chosen to model turbulence transport quantities: turbulence kinetic energy, $k$, and the specific dissipation rate, $\omega$. SST k- $\omega$ model was found to be one of the best performing RANS models in swirling and wall-bounded flows [16],[30][31].

The boundary conditions considered in this problem are mass flow inlet at the axial and tangential ports, pressure outlet at the nozzle exit plane and wall at the nozzle wall. The inlet conditions are adopted from the study [4] for realistic predictions. For no-swirl flow, axial flows (only) are provided from the bottom of the nozzle, with no flows from tangential ports. Conversely, for the swirling flows, three tangential ports supply the same amount of mass flow, with no axial flow from the bottom of the nozzle. This ensures the same Reynolds number, where the average velocity in Reynolds number is determined by volume flow rate divided by nozzle exit area. The pressure outlet condition is applied at the nozzle exit with atmospheric pressure whereby turbulence is specified by $2 \%$ intensity and hydraulic diameter. Finally, no-slip condition at the nozzle wall with ambient temperature is used.

\subsection{Numerical Settings and Model Validation}

The above governing equations (1-4) and two transport equations (SST k- $\omega$ ) are solved by finite volume based commercial software ANSYS Fluent v17. The pressure-based coupled algorithm is used to simultaneously solve the governing equations. For the pressure discretization PRESTO (PREssure STaggering Option) is applied, and the second-order central-differenced for diffusion terms and second-order upwind scheme for convective terms of the transport equations are used. Hexahedral mapped mesh type was used in multizone meshing with an element size $0.0025 \mathrm{~m}$. A typical mesh independence test is shown in Fig. 2 for 3TP. In this case, four different grid elements are tested, namely, 974001, 1310326, 1647089 and 1892870 elements. The results are found to be invariant, except the 974001 elements. As such, a mesh containing $1647 \mathrm{k}$ element is chosen in this study. Another testing is also done for first layer height on $\mathrm{y}^{+}$values and wall shear stress profiles, and is shown in Fig. 3. It appears the first layer cell height has little influence on those wall characteristics. As such, a first layer height of $0.05 \mathrm{~mm}$ is chosen in this study. The figure also shows that above settings ensured $\mathrm{y}^{+}$values less than 0.2 for the whole domain. The solution is assumed to be converged when the residuals of the flow parameters are less than $10^{-5}$. Mass conservation is also checked between inlet ports and the nozzle exit for converged solutions and the difference between inlets and outlet is found close to zero. Cartesian coordinates are given by $(x, y, z)$ with corresponding velocity components $\left(U_{x}, U_{y}, U_{z}\right)$. For convenience, results of this 3D simulation are presented by polar coordinates $(x, r, \theta)$ with $x$-axis coinciding for each system. In this regard, the corresponding axial $(U)$ and tangential $(W)$ velocity components are derived from the Cartesian coordinate data using the axes transformation rule.

\subsection{Parameter Definition}

The flow ratio $Q_{r}$ is the ratio of total mass flow rate through tangential inlets to the total flow rates in the nozzle, i.e.

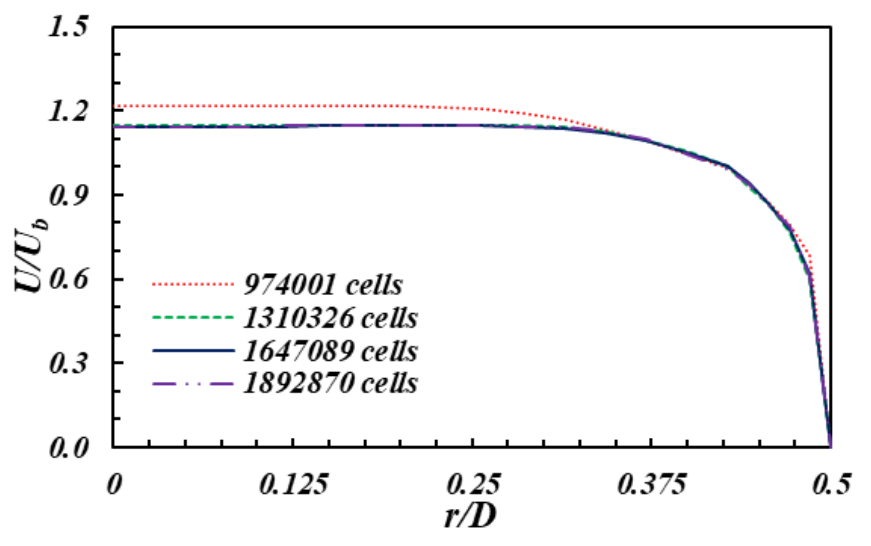

Fig. 2 Sensitivity of mesh elements for the case 3TP.

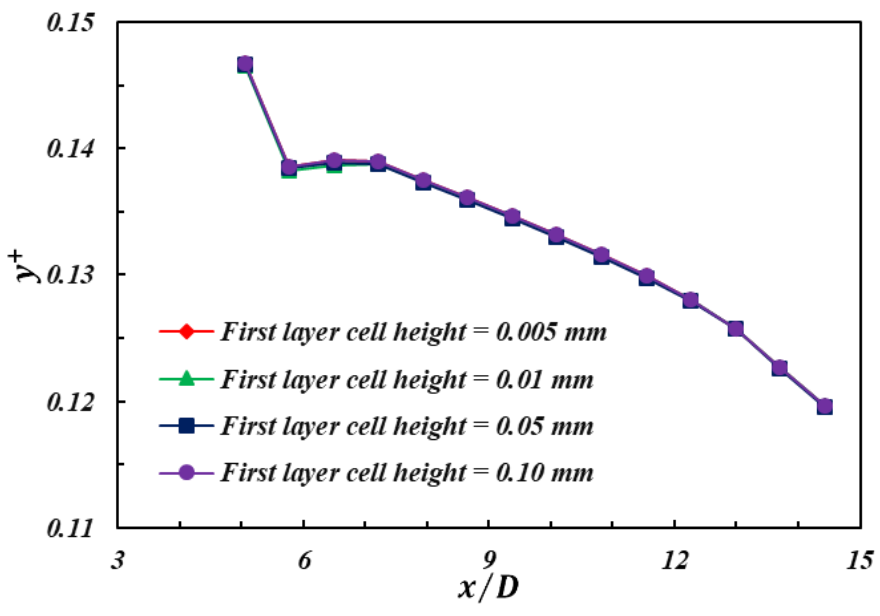

(a)

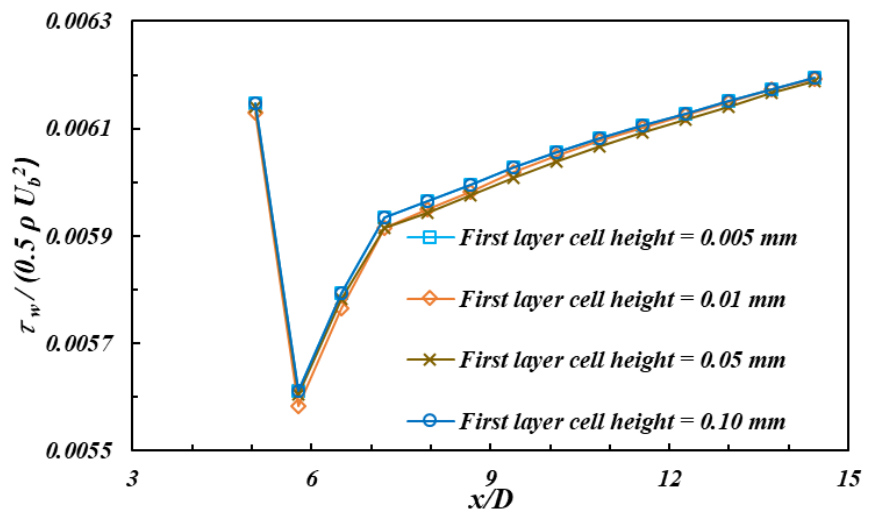

(b)

Fig. 3 Effect of first layer cell height on y+ and normalized wall shear stress.

sum of flowrates through all axial and tangential inlets. Mathematically,

$$
Q_{r}=\frac{Q_{t}}{Q_{a}+Q_{t}}=\frac{Q_{t}}{Q_{T}}
$$

The Reynolds number is defined as,

$$
\operatorname{Re}=\frac{Q_{T} D}{A v}
$$


Where, A is the nozzle area at the exit plane of the nozzle and $v$ is the dynamic viscosity of fluid. Two different swirl number definitions are common as the ratio of axial flux of tangential momentum to the axial flux of axial momentum:

$$
\begin{gathered}
S=\frac{2 \pi \rho \int_{0}^{R} r^{2} U W d r}{2 \pi R \rho \int_{0}^{R} r\left(U^{2}-0.5 W^{2}\right) d r} \\
S^{\prime}=\frac{\int_{0}^{R} r^{2} U W d r}{R \int_{0}^{R} r U^{2} d r}
\end{gathered}
$$

Finally, the local swirl number is defined as,

$$
S^{*}(r)=\frac{W}{U}
$$

\subsection{Validation}

The simulation data of the swirl nozzle is first tested with different turbulence models and validated by comparing with experimental data [4] at the nozzle outlet plane with three tangential ports for the case $Q_{r}=0$ and 1 (Fig. 4). It appears that SST $k-\omega$ model predicts the flow behavior well for both non-swirling and swirling cases. As such, SST $k$ - $\omega$ model will be used for the data presented in the ensuing results and discussion section. Fig. 4 also shows a good agreement between the numerical prediction and the experimental data for $Q_{r}=0$. A slight deviation is observed for $Q_{r}=1$, but importantly, the numerical data predicts well the profile behaviors and peak locations. The deviation is attributed to the possible measurement inaccuracy associated with CTA X-wire and experimental flow settings [13]. This results an overestimation of the mean velocity components than their corresponding true values. The deviations may also be partly attributed to the inability of RANS approach to accurately capture highly swirling flows.

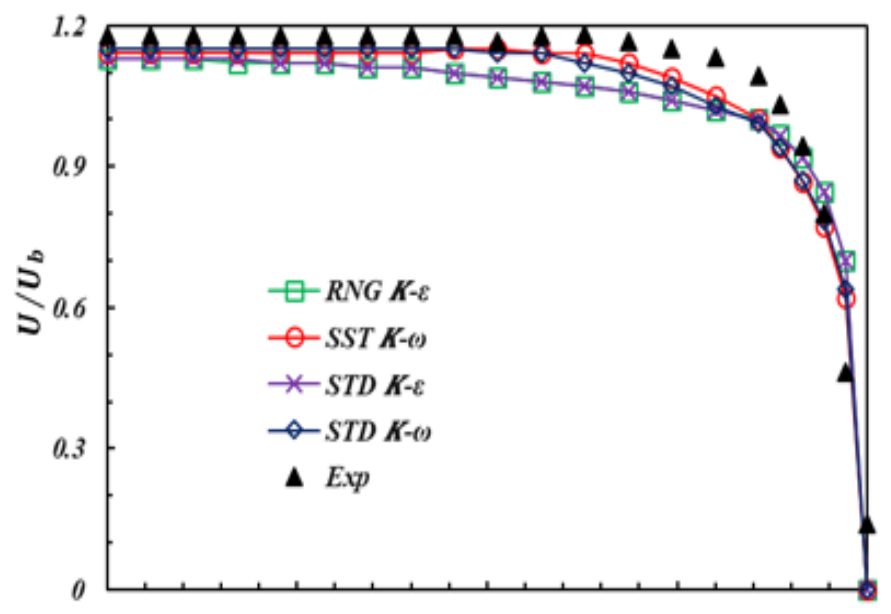

(a)

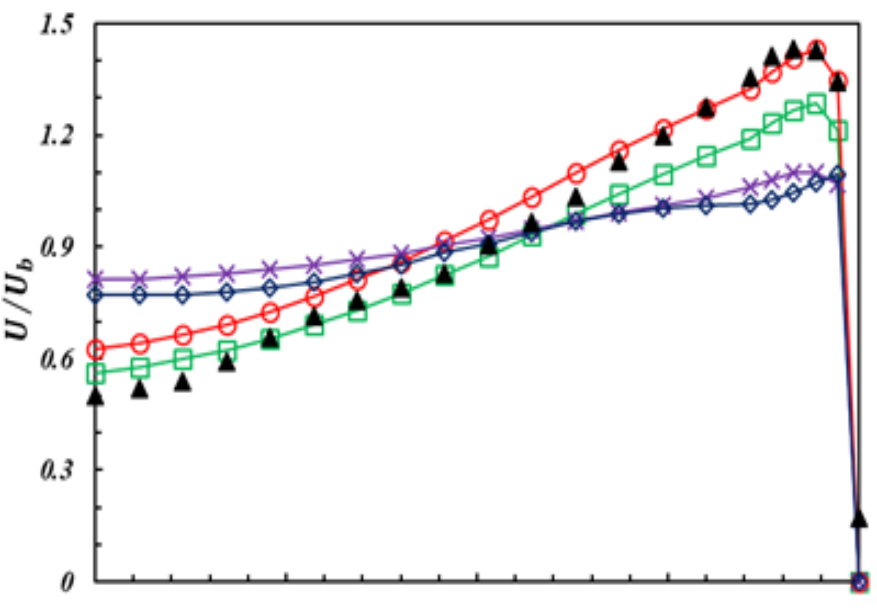

(b)

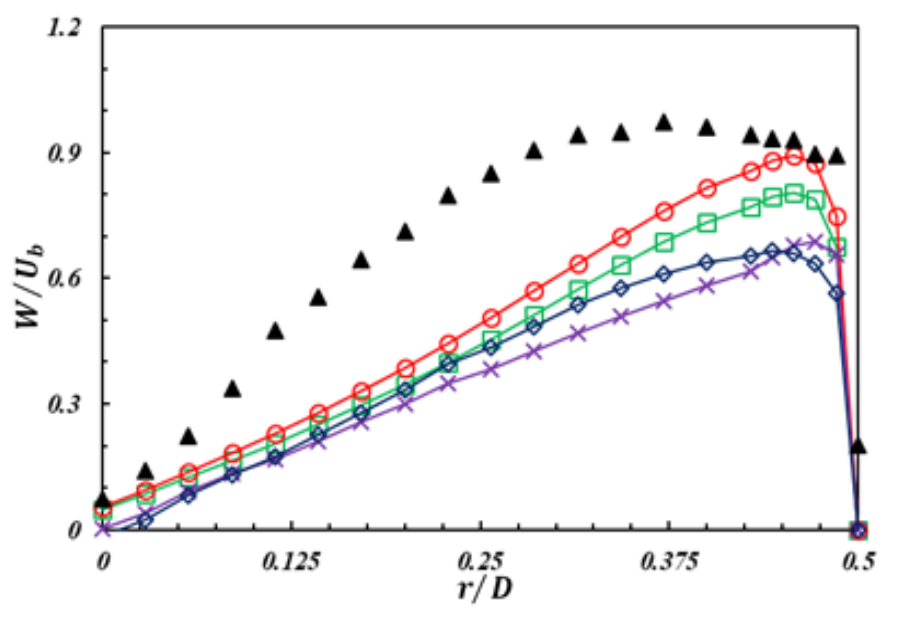

(c)

Fig. 4 Different turbulence models against experimental data [4] for (a) U/U_b for Q_r= 0, (b) U/U_b for Q_r= 1 and (c) W/U_b for Q_r $=1$.

\section{Results and Discussion}

This section includes presentation of mean and turbulence controlling parameters, such as velocity, boundary layer thickness, pressure drop, wall shear stress and turbulent shear stress for the effect of different number of tangential ports at two flow conditions $\left(Q_{r}=0\right.$ and $\left.Q_{r}=1\right)$.

Swirl decay along the length is shown in Fig. 5 for the 3TP case. The swirl number is calculated using the Equation (7) and Equation (8). It is observed that near the nozzle inlet the swirl number $S$ is highly fluctuating and after the converging section, the swirl number is nearly constant. Initially, the swirl number decreases from the inlet. When the tangential port has introduced the value of swirl number increases and immediately after the increment the swirl number drops again. In the nozzle converging section the magnitude of the swirl number rises again at first and then the value decreases and finally increases before coming at a constant magnitude. In case of $S^{\prime}$ the values initially increasing from the nozzle inlet come to a constant magnitude after the nozzle converging section. It is evident that the swirl number $S$ shows a very unpredictable nature before the nozzle converging section. 


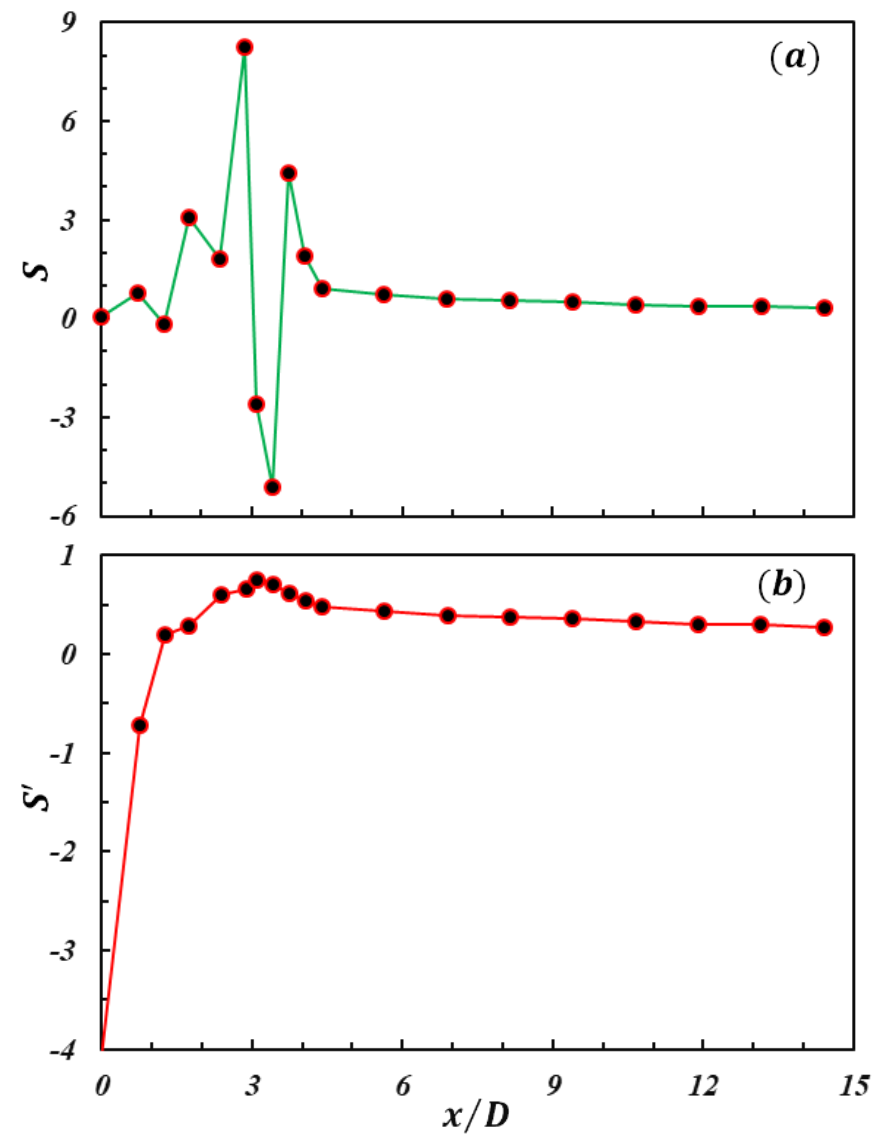

Fig. 5 Swirl decay along the length of the nozzle for 3TP case.

Fig. 6 shows the local swirl numbers along the radial location of the nozzle at the different axial positions $(x / D=$ $1.275,3.1,5.65,9.4$, and 14.425) of the nozzle for three different numbers of tangential ports, namely, 2TP, 3TP, and 4TP. It is observed that the profile of local swirl at $x / D=$ $5.65,9.4$, and 14.425 are almost the same in nature for all the cases. The local swirl increases gradually from the center of the nozzle toward the wall and near the wall, a sudden drop occurs. However, the local swirl profiles at $x / D=3.1$ are similar for 2TP and 3TP although for 4TP the local swirl profile is parabolic from the nozzle center toward the nozzle wall. The value of local swirl near the inlet at $x / D=1.275$ is highly unpredictable due to the swirl induction. The local swirl profile for 2TP and 3TP are nearly similar but for 4TP the profile is different. The local swirl near the nozzle wall suddenly increases and drops immediately after the increase and finally raise to the wall for both $2 \mathrm{TP}$ and $3 \mathrm{TP}$. However, the local swirl profile at 4TP is like a bell shape; increasing from the nozzle center it becomes constant at $r / D=0.2$ to $r / D=0.35$ and then decreases again towards the nozzle wall. As the swirl is introduced at this location the local swirl profile becomes highly unpredictable.

The axial velocity vectors at five different axial locations $(\mathrm{xD}=4.75,7.50,9.75,12.5$ and 14.425) are presented in Fig. 7 for non-swirling (Fig. 7a) and swirling (Fig. 7b-d) conditions. Velocity is found to be the almost uniform after the converging section for no swirl flow $\left(Q_{r}=0\right)$, but the magnitude is the highest near the exit plane, with a reduction towards the wall due to boundary layer formation. In contrast, for swirl flow $\left(Q_{r}=1\right)$, velocity magnitude is found to be the higher near the inlet and towards the nozzle exit, the velocity magnitude decreases. The axial velocity magnitude is proportional to the number of tangential ports and the velocity magnitude is zero at the wall due to no-slip condition. The axial velocity vectors at $x / D=4.75$ is symmetric from the centerline when swirl is induced (Fig. 7b-d), however, the velocity distribution becomes asymmetrical as the flow approaches the outlet. The velocity distribution is more symmetrical along the radial direction for 2TP than 3TP \& 4TP with a slight decrease near the center except for exit plane. At $x / D=14.425$, velocity reduction near the center is highest for 2TP.
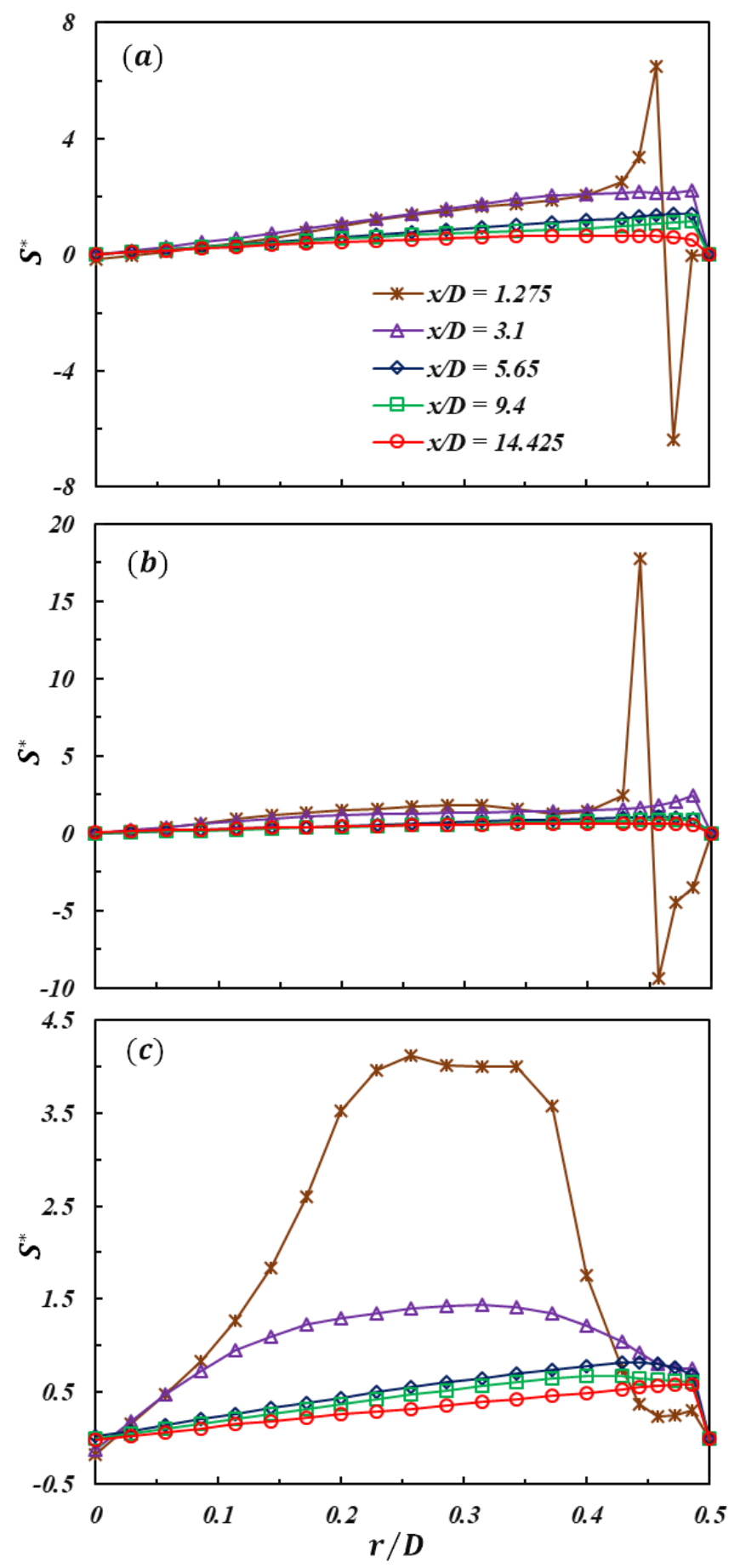

Fig. 6 Local swirl charactersitics along the length of the nozzle (a) 2TP, (b) 3TP, (c) 4TP. 


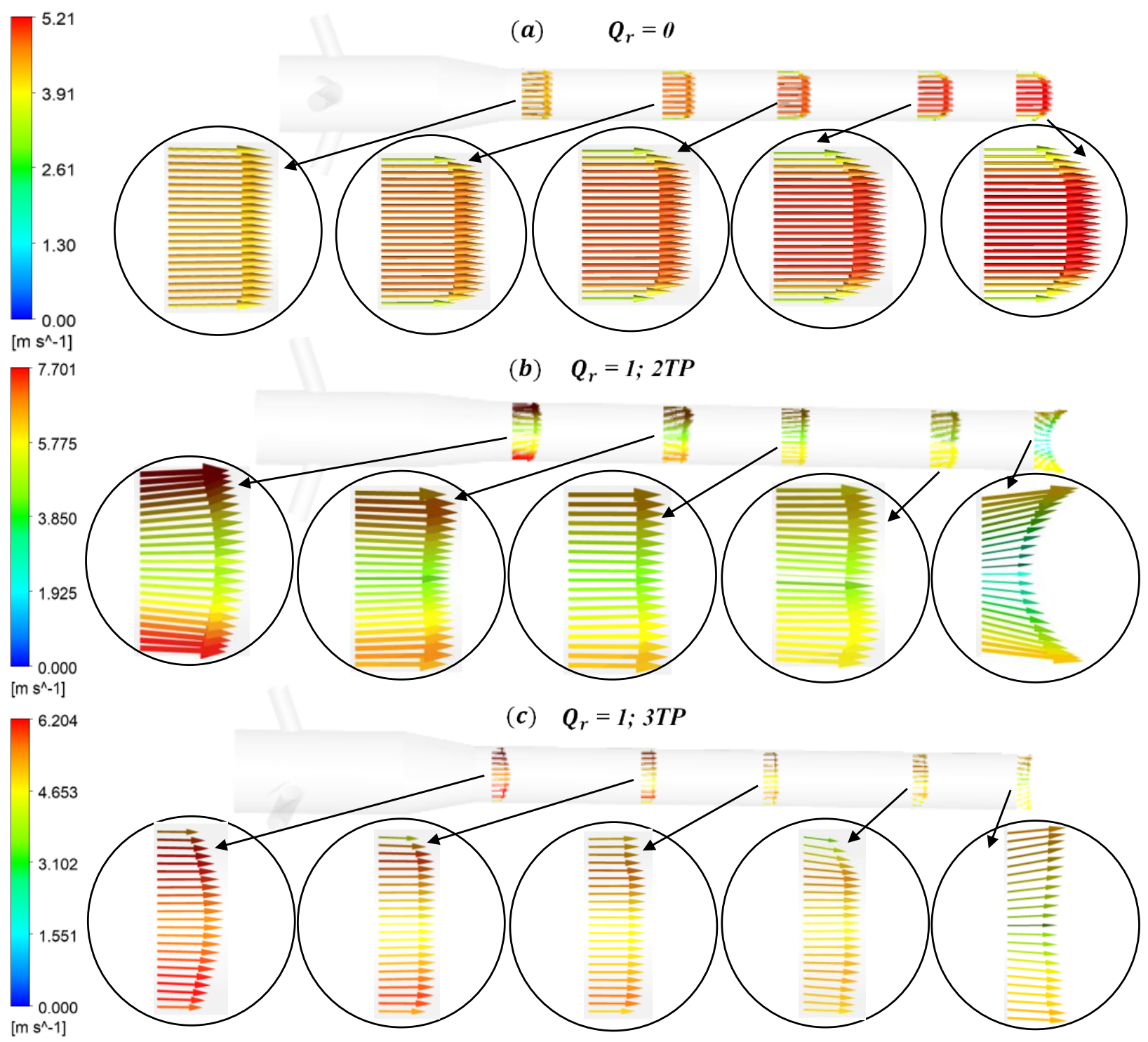

(d) $Q_{r}=1 ; 4 T P$

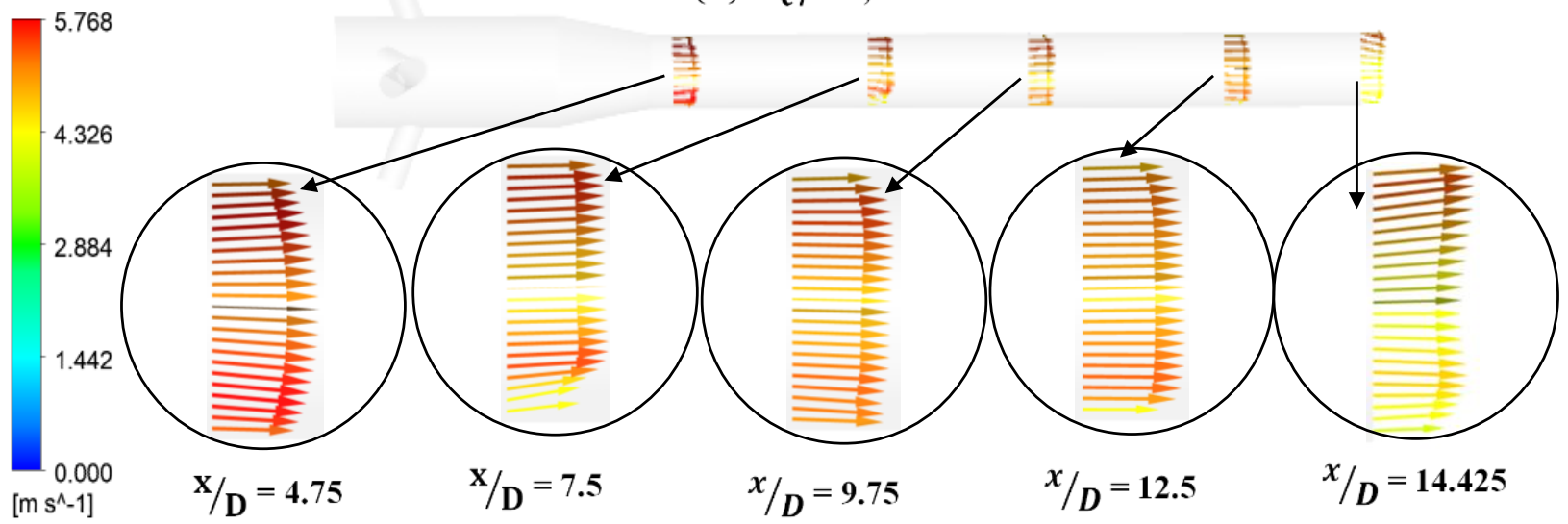

Fig. 7 Axial velocity vectors at different axial locations for non-swirling (a), and swirling flows: (b) 2TP, (c) 3TP and (d) 4TP. 
Fig. 8 depicts the boundary layer thicknesses along the length of the nozzle for both non-swirlign and swirling (2TP, 3TP and 4TP) conditions. It is observed that the magnitude of boundary layer thickness at 2TP is pretty low compare to the other. For swirl flow conditions the boundary layer thickness at the beginning is small and near the exit the boundary layer thickness increases. At the middle of the nozzle the boundary layer thickness is nearly constant. However, for no swirl flow condition the boundary layer thickness higher and then the boundary layer thickness gradually decreases towards nozzle exit.

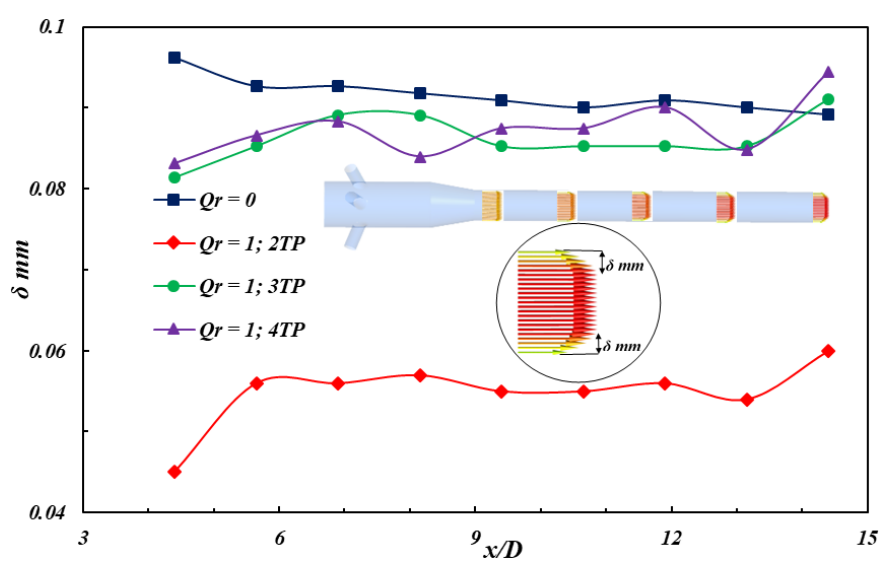

Fig. 8 Boundary layer thickness along the length of the nozzle.

Fig. 9 demonstrates the axial $\left(U / U_{b}\right)$ and tangential $\left(W / U_{b}\right)$ velocity components near the wall i.e. at $y / D=0.01,0.05$ and 0.1 for $Q_{r}=0$ and $Q_{r}=1$. In the figure, $Y$ represents the distance from the wall of the swirl nozzle. It is observed that at for swirl condition the near-wall axial velocity profile is identical for all the location and it increases from the inlet to the nozzle converging section. Then, the velocity suddenly decreases at $Y=0.01 D$ immediately after the converging section. The axial velocity is then nearly constant for all the axial location of the nozzle. However, the axial velocity decreases very little after the nozzle convergence at $Y=0.05 D$ and $0.1 D$, and no significant change occurs. For swirl flow condition the near-wall axial and tangential velocity increases after the swirl flow introduction. Then after the nozzle converging section the near-wall axial and tangential velocity show a very little deviation and along the nozzle axial location the velocity profile is nearly uniform at $Y=0.05 D$ and $Y=$ $0.1 D$. However, the near-wall axial and tangential velocity profile at $Y=0.01 D$ is fluctuates highly along the length of the nozzle, especially the tangential velocity profile shows a totally unpredictable nature. The overall near-wall axial and tangential velocity distribution at $Y=0.01 D$ is low in magnitude than at $Y=0.05 D$ and $Y=0.1 D$. It can be observed that the velocity profile at the wall vicinity $(Y=0.01 D)$ response highly than the other position.

The interplay between the number of tangential ports and the swirl intensity for the effect of skin friction coefficient along the length of the nozzle is shown in Fig. 10. It is observed that for no swirl condition the magnitude of Skin friction coefficient is very low and along the nozzle axial location no change occurs. However, the Skin friction coefficient at high swirl flow at $2 \mathrm{TP}$ is the maximum along the nozzle axial location although for 3TP and 4TP no significant deviation is observed. In every case of swirl flow the Skin friction coefficient at the nozzle exit is the maximum. Although the Skin friction coefficient along the axial location is not significantly changing however, near the exit it suddenly increase. The overall distribution of the Skin friction coefficient is not similar for all the case.

Fig. 11 displays the pressure drop at different radial locations for swirling flow (3TP). The inlet pressure is taken at the reference pressure and pressure drop is calculated based on the pressure of the nozzle inlet. It is observed that the pressure
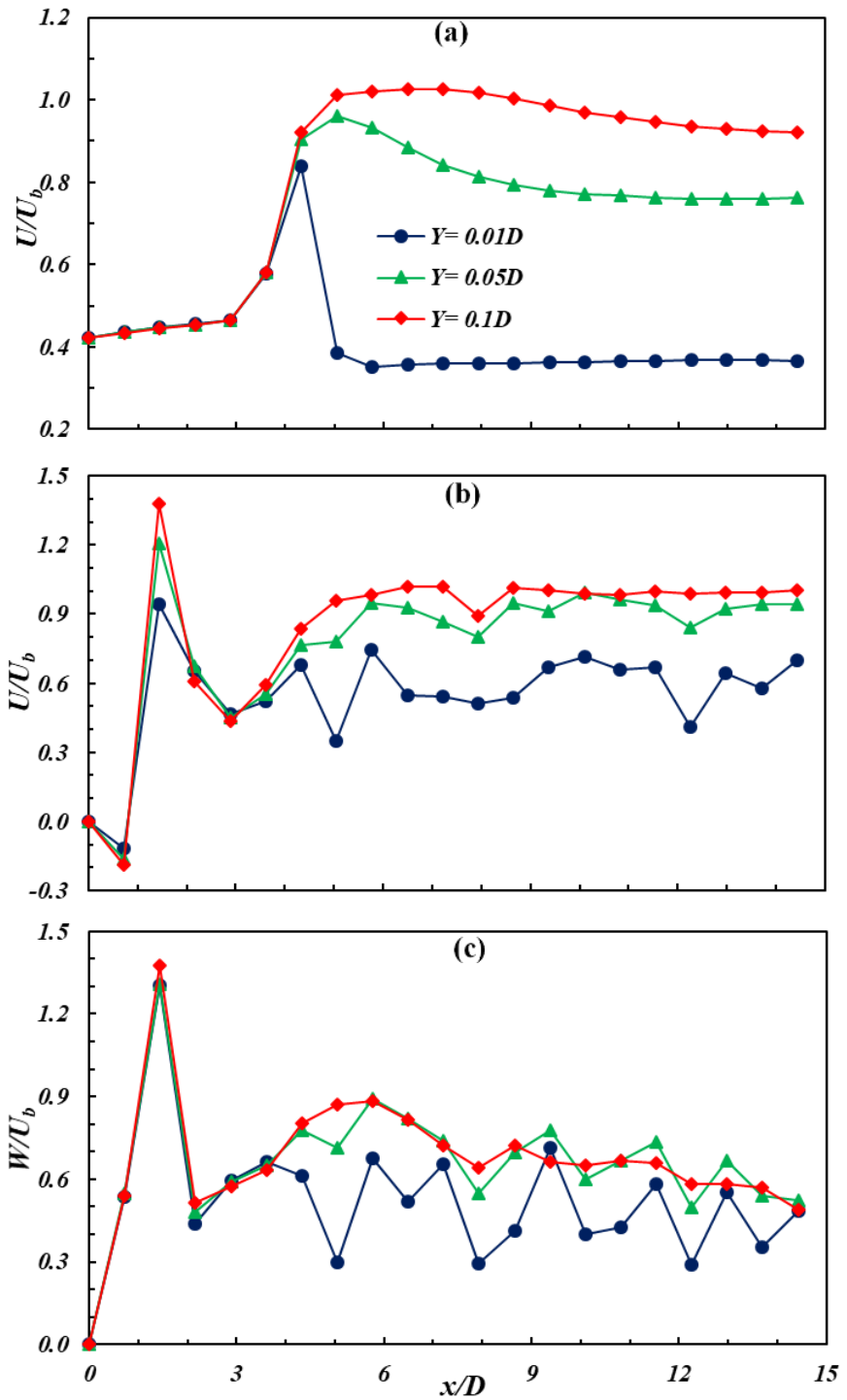

Fig. 9 Near-wall normalized axial $\left(\mathrm{U} / \mathrm{U}_{\mathrm{b}}\right)$ and tangential $\left(\mathrm{W} / \mathrm{U}_{\mathrm{b}}\right)$ velocity distrution along the nozzle length: $\mathrm{Q}_{\mathrm{r}}=0(\mathrm{a})$, and $\mathrm{Q}_{\mathrm{r}}=1$ (b and $\left.\mathrm{c}\right)$.

drop at the center of the nozzle $(r / D=0)$ is greater than all other radial location in almost every axial position of the nozzle, however, near the nozzle outlet it suddenly reduces. While pressure drop at every radial location increase after the introduction of the tangential ports, at $(r / D=0.4)$ it suddenly decrease. Pressure drop near the wall is gradually increasing after the converging section of nozzle but pressure drop near the nozzle center is nearly constant. It can be concluded that Due to the tangential flow introduction the pressure drop characteristics near the nozzle center response quickly and near the wall vicinity this property change slowly. 


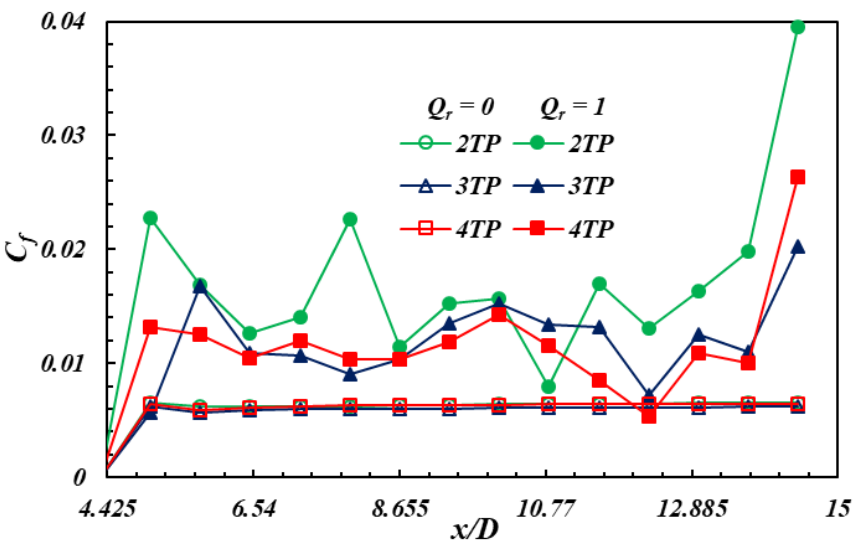

Fig. 10 Skin friction coefficient along the nozzle length.

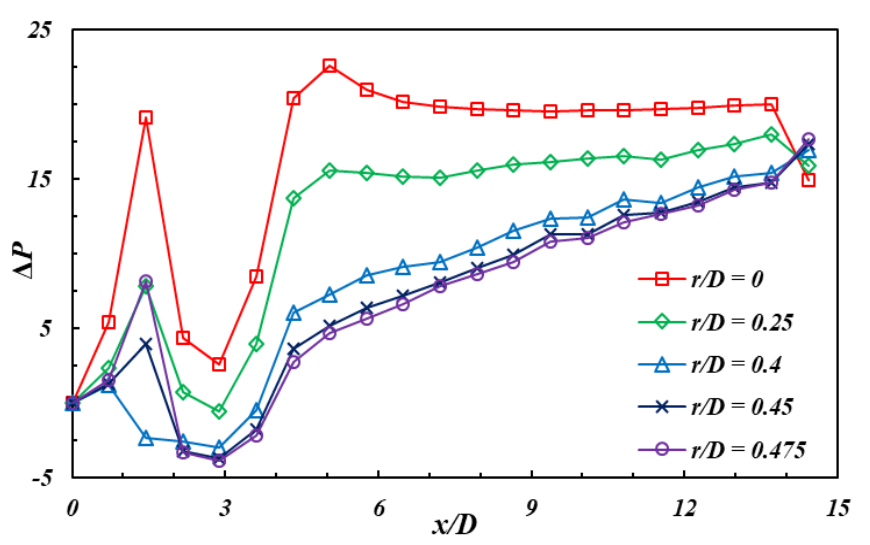

Fig. 11 Pressure drop along the nozzle length for swirling flow.

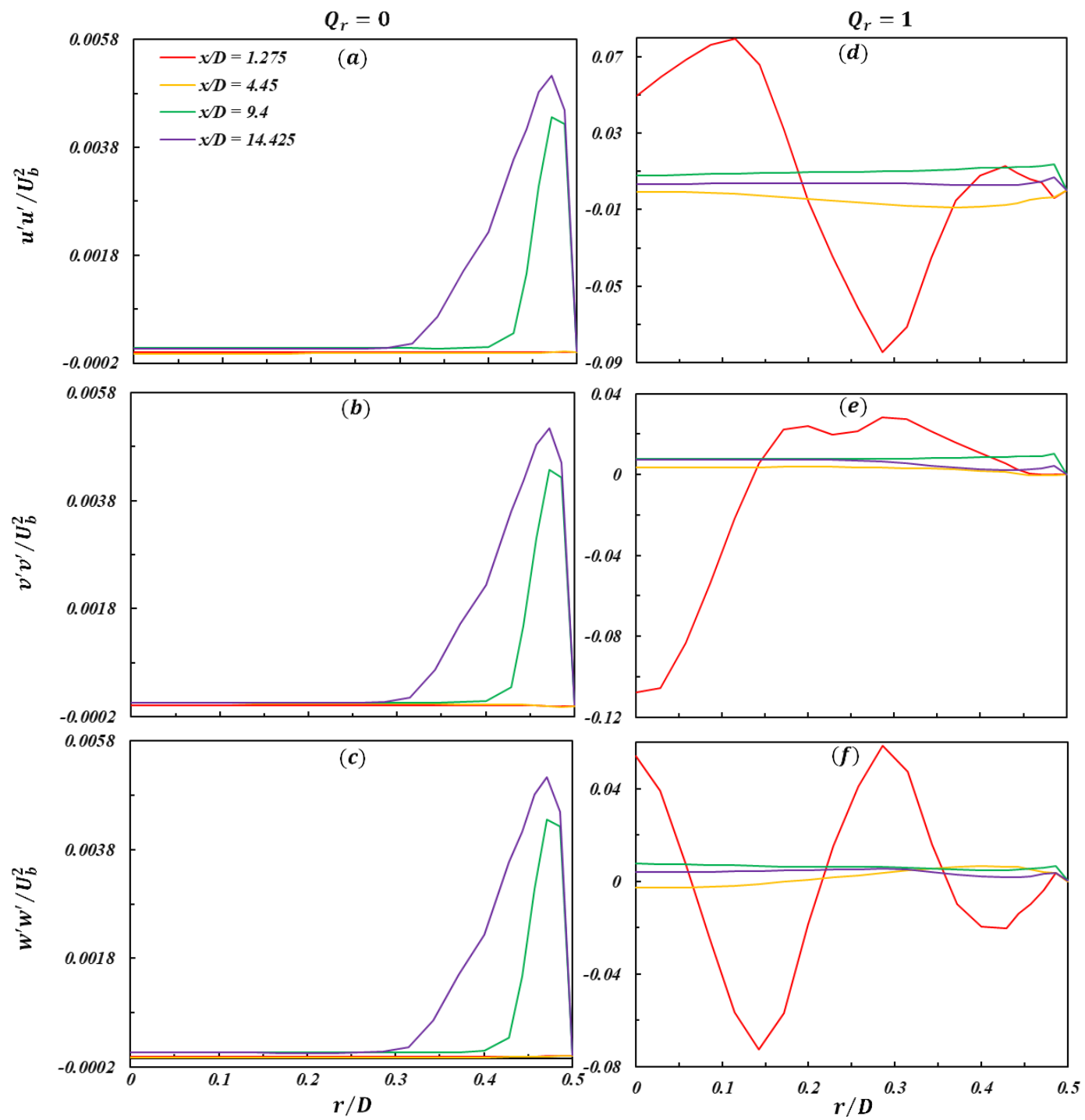

Fig. 12 Turbulent normal stress profiles at different axial locations.

Fig. 12 presents the radial distribution of normalized turbulent normal stress components at various axial locations. It is observed that the turbulent flow characteristic near the wall is very high at $x / D=9.4$ and $x / D=14.425$ at no swirl flow $\left(Q_{r}=0\right)$ although at the center of the nozzle the turbulent flow characteristic is very low. Moreover, near the inlet of the nozzle at $x / D=1.275$ and $x / D=4.45$ the overall turbulent flow characteristic is very small in magnitude. On the contrary at swirl flow condition $\left(Q_{r}=1\right)$ near the inlet of the nozzle at $x / D=1.275$ the overall turbulent flow characteristic is very high. However, the turbulent normal stress near the outlet section at $x / D=9.4$ and $x / D=14.425$ is small in these 
locations. While at no swirl condition the turbulent normal stress characteristic at the center of nozzle is very low at swirl flow condition, the $u^{\prime} u^{\prime}$ and $w^{\prime} w^{\prime}$ component is very high at the nozzle center especially at $x / D=1.275$ due to the

$$
Q_{r}=0
$$

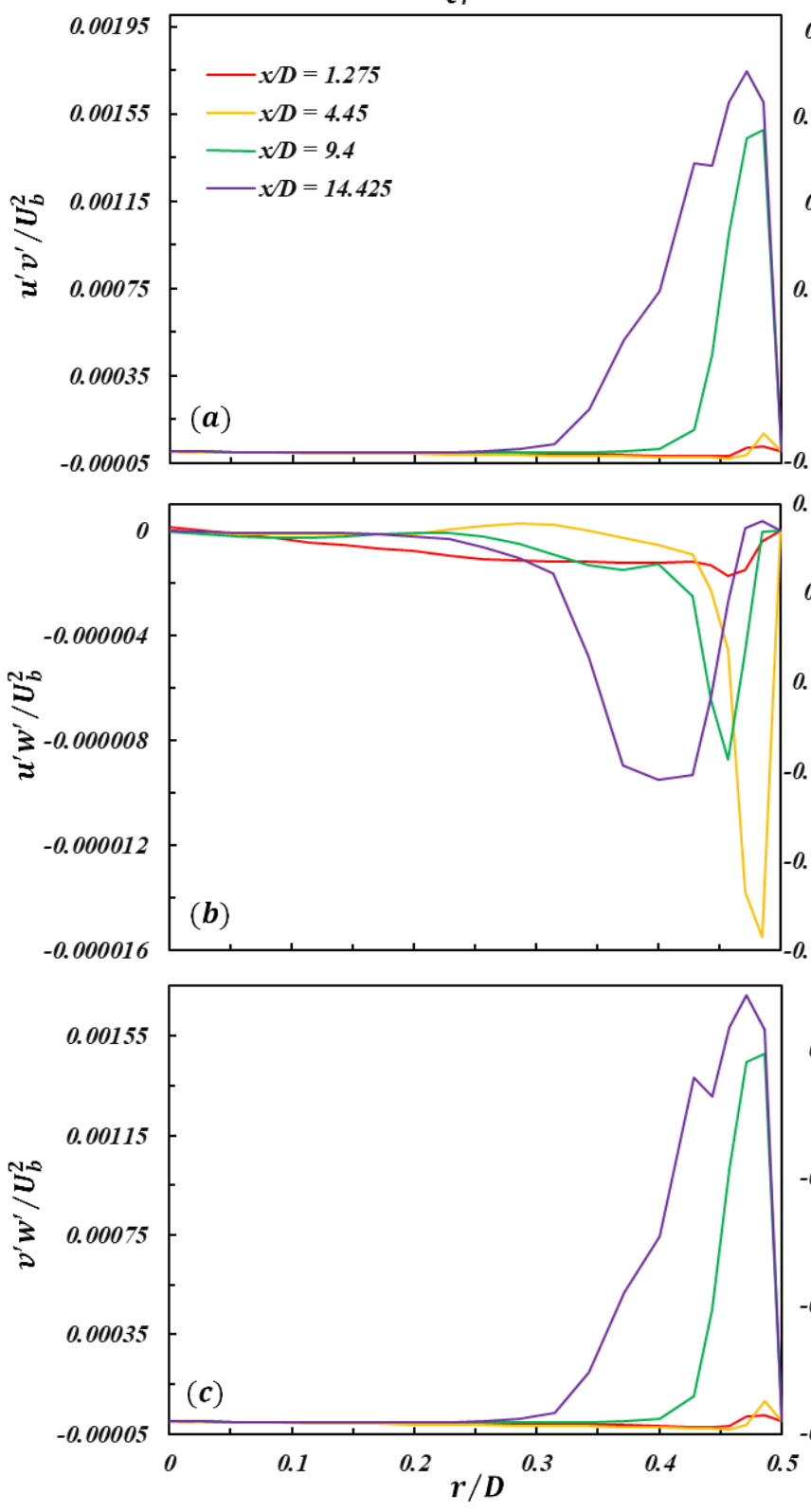

introduction of swirl flow. The overall magnitude of the turbulent normal stress is very small in no swirl flow condition but for swirl condition it is very high.
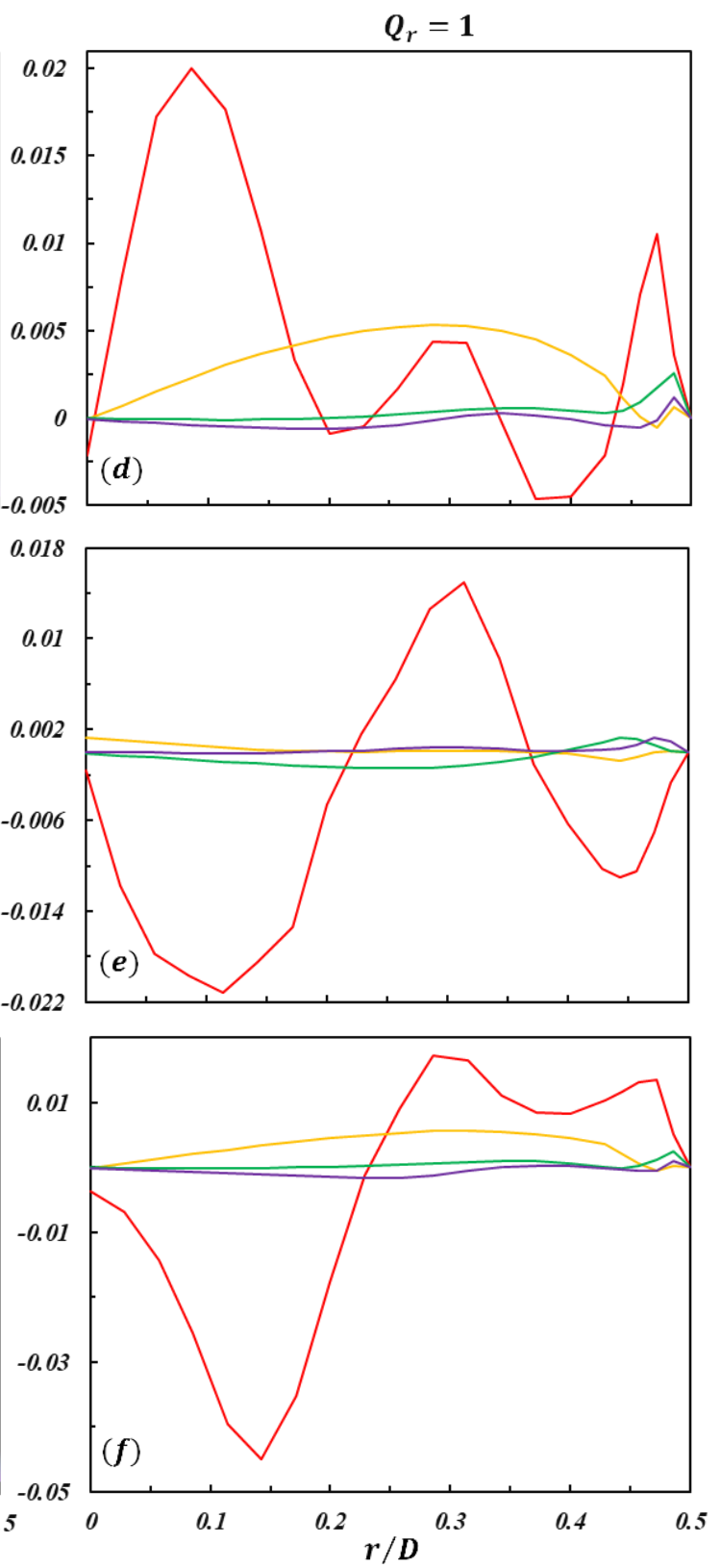

Fig. 13 Turbulent shear stress profiles at different axial locations.

Finally, Fig. 13 presents the radial distribution of normalized turbulent shear stress components at various axial locations. It is observed that for no swirl flow $\left(Q_{r}=0\right)$ the turbulent shear stress component $u^{\prime} v^{\prime}$ and $v^{\prime} w^{\prime}$ near the wall is very high at $x / D=9.4$ and $x / D=14.425$ although at the center of the nozzle the turbulent share stress component is very low (Fig. 13a and Fig. 13c). However, the $u^{\prime} w^{\prime}$ component at $x / D=4.45,9.4$ and 14.425 is reducing near the wall of the nozzle. Near the inlet of the nozzle at $x / D=1.275$ the overall turbulent share stress is uniform and does not deviate much for no swirl flow $\left(Q_{r}=0\right)$. On the other hand at swirl flow condition $\left(Q_{r}=1\right)$ near the inlet of the nozzle at $x / D=1.275$ all the three component of turbulent share stress is fluctuates highly and the magnitude of overall turbulent share stress is very high. However, the overall turbulent share stress at $x / D=$ 9.4 and $x / D=14.425$ is very uniform along the radial location for swirl flow. The overall magnitude of the turbulent flow characteristic is very small in for swirl flow compared to the swirl condition.

\section{Conclusion}

An incompressible turbulent swirling air jet is investigated numerically. In this regard, an axial-plus-tangential swirl flow is considered for non-swirling $\left(\mathrm{Q}_{\mathrm{r}}=0\right)$ and highly swirling $\left(Q_{r}=1\right)$ cases. Finite volume based commercial software ANSYS Fluent v17 is used in the simulation to investigate mean flow and turbulence characteristics. Governing equations are approximated by the RANS equations and turbulence is 
characterized by the SST $k-\omega$ model. The study examines the effect of number of tangential inlets on mean flow behaviors and turbulent characteristics. The magnitude of swirl decay is fluctuates highly before the nozzle converging section and after the nozzle converging section the swirl decay is nearly constant. The boundary layer thickness in swirl flow is found to be the smallest for 2TP. The skin friction coefficient along the nozzle axial position at no swirl flow is uniform and the magnitude is very small. For swirl flow, the skin friction coefficient along the nozzle axial position is fluctuating especially for 2TP. Moreover, the skin friction coefficient at the nozzle exit is the maximum for all the case. Due to the tangential flow introduction, the pressure drop near the nozzle center response quickly and near the wall vicinity this property changes slowly. The turbulent normal and shear stress for no swirl flow is nearly uniform, but for swirl flow it fluctuates the most near the section where the swirl flow is introduced.

\section{Acknowledgements}

The first author acknowledges the Department of Electronics and Communication Engineering at Khulna University of Engineering \& Technology, Bangladesh for part of the computation facility provided during the study program.

\section{References}

[1] Sloan, D.G., Smith, P.J. and Smoot, L.D., 1986. Modeling of swirl in turbulent flow systems. Progress in Energy and Combustion Science, 12(3), pp.163-250.

[2] Rose, W.G., 1962. A swirling round turbulent jet, Journal of Applied Mechanics, vol.29, Trans. ASME, vol. 84, Series E, pp. 616-625.

[3] Toh, K., Honnery, D. and Soria, J., 2010. Axial plus tangential entry swirling jet. Experiments in Fluids, 48(2), pp.309-325.

[4] Ahmed, Z.U., 2016. An experimental and numerical study of surface interactions in turbulent swirling jets. $\mathrm{PhD}$ Thesis, Edith Cowan University, Australia.

[5] Gore, R.W. and Ranz, W.E., 1964. Backflows in rotating fluids moving axially through expanding cross sections. AIChE Journal, 10(1), pp.83-88.

[6] Jafari, M., Farhadi, M. and Sedighi, K., 2017. An experimental study on the effects of a new swirl generator on thermal performance of a circular tube. International Communications in Heat and Mass Transfer, 87, pp.277-287.

[7] Markal, B., 2018. Experimental investigation of heat transfer characteristics and wall pressure distribution of swirling coaxial confined impinging air jets. International Journal of Heat and Mass Transfer, 124, pp.517-532.

[8] Yajnik, K.S. and Subbaiah, M.V., 1973. Experiments on swirling turbulent flows. Part 1. Similarity in swirling flows. Journal of Fluid Mechanics, 60(4), pp.665-687.

[9] Chang, F. and Dhir, V.K., 1994. Turbulent flow field in tangentially injected swirl flows in tubes. International journal of heat and fluid flow, 15(5), pp.346-356.

[10] KITO, O. and KATO, T., 1984. Near wall velocity distribution of turbulent swirling flow in circular pipe. Bulletin of JSME, 27(230), pp.1659-1666.

[11] Kitoh, O., 1991. Experimental study of turbulent swirling flow in a straight pipe. Journal of Fluid Mechanics, 225, pp.445-479.

[12] Buschmann, M.H., Indinger, T. and Gad-el-Hak, M., 2009. Nearwall behavior of turbulent wall-bounded flows. International Journal of Heat and Fluid Flow, 30(5), pp.993-1006.

[13] Ahmed, Z.U., Al-Abdeli, Y.M. and Guzzomi, F.G., 2016. Corrections of dual-wire CTA data in turbulent swirling and nonswirling jets. Experimental Thermal and Fluid Science, 70, pp.166-175.
[14] Ahmed, Z.U., Khayat, R.E., Maissa, P. and Mathis, C., 2012. Axisymmetric annular curtain stability. Fluid Dynamics Research, 44(3), p.031401:1-23.

[15] Ahmed, Z.U., Khayat, R.E., Maissa, P. and Mathis, C., 2013. Non-axisymmetric annular curtain stability. Physics of Fluids, 25(8), p.082104:1-37.

[16]Lu, P. and Semião, V., 2003. A new second-moment closure approach for turbulent swirling confined flows. International journal for numerical methods in fluids, 41(2), pp.133-150.

[17] Tsai, J.H., Lin, C.A. and Lu, C.M., 1995. Modelling dump combustor flows with and without swirl at the inlet using Reynolds stress models. International Journal of Numerical Methods for Heat \& Fluid Flow, vol. 5, no. 7, pp. 577-588.

[18] Gorman, J.M., Sparrow, E.M., Abraham, J.P. and Minkowycz, W.J., 2016. Evaluation of the efficacy of turbulence models for swirling flows and the effect of turbulence intensity on heat transfer. Numerical Heat Transfer, Part B: Fundamentals, 70(6), pp.485-502.

[19] Saqr, K.M. and Wahid, M.A., 2014. Effects of swirl intensity on heat transfer and entropy generation in turbulent decaying swirl flow. Applied thermal engineering, 70(1), pp.486-493.

[20] Nouri-Borujerdi, A. and Kebriaee, A., 2012. Simulation of turbulent swirling flow in convergent nozzles. Scientia Iranica, 19(2), pp.258-265.

[21] Najafi, A.F., Saidi, M.H., Sadeghipour, M.S. and Souhar, M., 2005. Numerical analysis of turbulent swirling decay pipe flow. International communications in heat and mass transfer, 32(5), pp.627-638.

[22] Islam, Md. M., Tasnim, S., and Ahmed, Z. U., 2017. Numerical study of a swirl nozzle at moderate swirl number, International Conference on Mechanical Engineering and Renewable Energy, 20-22 December, Chittagong, Bangladesh.

[23] Islam, S. M, Khan, M. T., Ahmed, Z. U., 2020. Effect of design parameters on flow characteristics of an aerodynamic swirl nozzle, Progress in Computational Fluid Dynamics, In press.

[24] Khan, M. H. U., and Ahmed, Z. U., 2019. Fluid flow and heat transfer characteristics of multiple swirling impinging jets at various impingement distances, International Journal of Thermofluid Science and Technology, vol. 6, no. 4, pp. 19060403:1-12.

[25] Ahmed, Z. U., Al-Abdeli, Y. M., 2017. Flow characteristics due to jet impact at low intensity, In: Proceedings of Int. Conf. Engineering, Research, Innovation and Education, Sylhet, Bangladesh, 156, p. 1-6.

[26] Debnath, S., Khan, M.H.U. and Ahmed, Z.U., 2020. Turbulent Swirling Impinging Jet Arrays: A Numerical Study on Fluid Flow and Heat Transfer. Thermal Science and Engineering Progress, p. 100580 .

[27] Ahmed, Z.U., Khan, M.H.U., Khayat, R.E. and Tasnim, S., 2018, July. Effect of flow confinement on the hydrodynamics and heat transfer characteristics of swirling impinging jets. In AIP Conference Proceedings (Vol. 1980, No. 1, p. 040008). AIP Publishing LLC.

[28] Thomas, B.K., Ahmed, Z.U., Al-Abdeli, Y.M. and Matthews, M.T., 2013. The optimisation of a turbulent swirl nozzle using CFD. Proceedings of the Australian Combustion Symposium, November 6-8, Perth, Australia.

[29] Menter, F.R. and Egorov, Y., 2010. The scale-adaptive simulation method for unsteady turbulent flow predictions. Part 1: theory and model description. Flow, Turbulence and Combustion, 85(1), pp.113-138.

[30] Ahmed, Z.U., Al-Abdeli, Y.M. and Matthews, M.T., 2015. The effect of inflow conditions on the development of non-swirling versus swirling impinging turbulent jets. Computers \& Fluids, 118, pp.255-273.

[31] Ahmed, Z.U., Al-Abdeli, Y.M. and Guzzomi, F.G., 2017. Flow field and thermal behaviour in swirling and non-swirling turbulent impinging jets. International Journal of Thermal Sciences, 114, pp.241-256. 